ESJ Social Sciences

\title{
La stabilité financière des Systèmes Financiers Décentralisés (SFD) au Sénégal : quelle situation après une nouvelle régulation prudentielle?
}

\section{Dr. Cheikh Mbacké Diop}

Maître assistant à l'Université Alioune DIOP (UAD) de Bambey (Sénégal), chef de département de management, membre de l'Equipe de Recherche en Innovation Managériale (ERIM-UADB) et du laboratoire Finance

Organisation Contrôle et Stratégie (FOCS-UCAD)

Doi:10.19044/esj.2021.v17n1p146

Submitted: 04 November 2020

Accepted: 25 December 2020

Published: 31 January 2021
Copyright 2021 Author(s)

Under Creative Commons BY-NC-ND

4.0 OPEN ACCESS

Cite As:

Diop M.C. (2021). La stabilité financière des Systèmes Financiers Décentralisés (SFD) au Sénégal : quelle situation après une nouvelle régulation prudentielle ?. European Scientific Journal, ESJ, 17(1), 146. https://doi.org/10.19044/esj.2021.v17n1p146

\section{Résumé}

Ce travail cherche à analyser la stabilité financière des SFD au Sénégal à travers la solidité financière et la protection des épargnants, suite à l'application d'une nouvelle régulation prudentielle en 2010. La méthodologie repose sur une base de données de panel de 42 SFD de 2010 à 2015. Elle est consolidée et mise à notre disposition par la direction de la réglementation qui assure la tutelle des SFD. Un traitement de normalisation par la méthode « Min-Max », suivi d'une pondération et d'une agrégation par une analyse en composante principale (ACP) ont permis de construire un indice agrégé de stabilité et des sous indices pour apprécier la stabilité financière. Les résultats soulignent une stabilité financière et de ses dimensions (rentabilité, qualité des actifs et protection des actifs). Cependant, des efforts supplémentaires permettraient de hisser les niveaux de rentabilité, de diminuer le PAR et de corriger la tendance baissière des indicateurs de la protection des épargnants. La disponibilité des données, au-delà de 2015, serait une valeur ajoutée. Il serait important aussi de mieux analyser les déterminants de la liquidité de 1'actif qui contribue à hauteur de $40 \%$ à la protection des épargnants. 
Mots clés : Stabilité financière, Régulation prudentielle, Systèmes Financiers Décentralisés

\title{
Financial Stability of Decentralized Financial System in Senegal: What Situation After a New Prudential Regulation?
}

\author{
Dr. Cheikh Mbacké Diop
}

Maître assistant à l'Université Alioune DIOP (UAD) de Bambey (Sénégal), chef de département de management, membre de l'Equipe de Recherche en Innovation Managériale (ERIM-UADB) et du laboratoire Finance

Organisation Contrôle et Stratégie (FOCS-UCAD)

\begin{abstract}
This work seeks to analyze the financial stability of the Decentralized Financial System(DFS) in Senegal through the financial robustness and protections of savers, after the application of new prudential regulation in 2010. The methodology consists of a database of 42 Decentralized Financial System panels from 2010 to 2015. It is consolidated and made available at our disposal by the Direction of the regulation which assures the guardianship of DFSs. A regulation by the method «Min-Max », followed by a ponderation and aggregation by a component analysis have permitted to build an aggregated index of stability and sub-indexes to give an appreciation of the financial stability. The results underline financial stability and its dimensions (profitability, quality of assets and assets protection). However, supplementary efforts should be permitted to boost the level of rentability, to increase the PAR and to correct decreased tendency of indicators of the protection of savers. The availability of datas, beyond 2015, should be an added value. It also should be important to better analyze the determinants of asset quality which contributes to the level of $40 \%$ to the protection of savers.
\end{abstract}

Keywords: Financial stability, prudential regulation, decentralized financial systems 


\section{Introduction}

Après les années 2000, le secteur de la microfinance s'est essoufflé avec des difficultés notées au niveau de plusieurs SFD. Une catégorie d'acteurs assez significatifs du dispositif de financement comme les groupements d'épargne et de crédit (GEC) et les structures signataires de convention cadre (SSCC) montrent leurs limites en termes de viabilité et de potentiel économique. Une bonne partie des mutuelles d'épargne ont également rencontré ces mêmes difficultés et des problèmes importants de qualité du portefeuille de crédit sont constatés (Sakho, 2004 ; BCEAO, 2001). Ces difficultés, recensées à une période d'émergence de nouveaux types de SFD de forme sociétaire, conduisent à l'adoption de la loi n²008-47 et des ratios prudentiels de la BCEAO.

Cette loi et ces ratios entrent en vigueur à partir de 2010 et permettent un assainissement du secteur à travers un régime unique d'autorisation d'exercice à tous les SFD, la suppression des GEC, des SSCC et des mutuelles n'ayant pas une capacité de résilience.

L'assainissement permet de protéger les acteurs qui déposent leurs ressources financières afin de leur éviter les conséquences de la défaillance ou de la faillite de SFD non viables.

Cette loi, poursuit aussi un objectif de stabilisation du secteur de la microfinance avec l'implication de la banque centrale dans le resserrement des conditions d'entrée et l'instruction des dossiers d'autorisation d'exercice. Également, la stabilisation du secteur de la microfinance demande le renforcement de la supervision de la BCEAO et de la DRS.

Le respect des dispositions de surveillance permettrait d'avoir des SFD solides, viables et capables de dérouler les activités d'épargne et de crédit sur une longue période (Hoxhaj, 2010). Il permet de protéger les clients épargnants en anticipant sur les risques de solvabilité, de liquidité etc. En Afrique centrale, plusieurs dispositions prudentielles ne sont pas respectées et mettent les SFD dans une situation de fragilité, au regard de plusieurs normes de solidité. Une bonne partie des SFD ne respectent pas les taux de participation. Pour certains, les fonds propres sont insuffisants. Ces deux situations sont synonymes de manque de solvabilité et exposent les clients à difficultés de retrait (BEAC, 2016).

La surveillance du secteur financier élargit les pouvoirs des organes de régulation, réduit les pratiques abusives et les prises de risques excessifs (OCDE, 2010). La gestion des risques crée de la valeur, de la rentabilité et rend performant les SFD à travers un dispositif de régulation prudentielle (Tchuigoua et Nekhili, 2012).

La réglementation et la supervision SFD, à travers une loi et des indicateurs à atteindre, constituent les piliers d'une régulation prudentielle destinée à rendre solide les SFD et à protéger les épargnants. Cette solidité 
financière, synonyme de performance financière, est mesurée par les indicateurs de qualité de portefeuille, de rendement, d'autosuffisance opérationnelle etc. (Ellé, 2017).

$\mathrm{Au}$ regard des objectifs d'une régulation prudentielle, la question suivante est adressée :

Après l'entrée en vigueur d'une nouvelle régulation prudentielle ${ }^{1}$ en 2010, les SFD au Sénégal ont-ils bénéficié d'une stabilité financière renforcée?

Il s'agira de construire un indice agrégé de stabilité financière (IASF) pour analyser l'évolution de la situation sur la période post réglementation. Il sera aussi question de voir, de façon spécifique, la dynamique observée en termes de solidité financière et de protection des clients épargnants. Ainsi, les questions subsidiaires sont formulées:

- La solidité financière des SFD s'est-elle améliorée ?

- Les épargnants bénéficient-ils d'une bonne protection?

- Avec un IASF, constate-t-on une amélioration de la stabilité financière des SFD ?

Le travail a pour objet d'analyser la stabilité financière des SFD après des années de réforme du cadre juridique pour une amélioration et une pérennité des activités de microfinance.

Les objectifs spécifiques permettant de mieux faire l'analyse se résument cidessous :

- Vérifier si les SFD bénéficient d'une solidité financière ;

- Analyser la protection dont bénéficient les épargnants ;

- Construire un indice agrégé et analyser la stabilité financière globale des SFD.

La méthodologie retenue consiste à utiliser des données de panel sur la période post réforme et surtout à partir de 2010 où la fourniture d'informations sur ces indicateurs avait démarré. Des analyses tendancielles sont faites à partir des composantes de la solidité financière et de la protection des clients. Aussi, une tendance globale de la stabilité financière est présentée à partir des résultats de l'IASF.

Ce travail se répartit en trois parties: une revue conceptuelle et théorique sur la régulation prudentielle et la stabilité financière (partie I), la méthodologie de construction d'indice agrégé (partie II) et la présentation des résultats (partie III).

${ }^{1}$ Elle fait référence à la loi n ${ }^{\circ} 2008-47$ et aux ratios prudentiels de la BCEAO de 2010 


\section{Corpus théorique sur la régulation prudentielle et de la stabilité financière}

La régulation prudentielle a toujours une finalité de performance et de protection à travers un dispositif, souvent coercitif, de réglementation et de supervision.

\section{I.1. Utilité d'une régulation prudentielle coercitive sur la stabilité financière}

La régulation est, en partie, un dispositif de gouvernance externe, permettant, suivant une approche organisationnelle, de combler les défaillances de contrôle interne, de protéger les clients, de générer des bénéfices marginaux sur la base d'un couple bénéfice/coût (Tchuigoua et Nekhili, 2012). Le dispositif mis en place permet de couvrir les clientsépargnants contre les comportements opportunistes des dirigeants et des managers. Les règles du jeu sont clairement fixées et le suivi de l'exploitation est fait au profit des clients qui, du fait de l'opacité organisationnelle, ne parviennent pas à obtenir les bonnes informations.

Dans des contextes de microfinance où il y a une forte concentration de coopératives ou de mutuelles, il est constaté que les sociétaires ont une faible implication dans le contrôle de la gestion. Ils s'intéressent plus à des avantages sur la facturation des crédits obtenus et des épargnes constituées. Cette situation donne plus de latitude aux dirigeants qui pourront s'accaparer des rentes créées, à l'absence de régulation et d'organe de contrôle externe (Ory, Jaeger et Gurtner, 2006).

Aussi, dans une situation de collecte de l'épargne publique, des règles prudentielles doivent être adoptées afin de protéger les épargnants en leur garantissant un retrait des dépôts, tributaire d'une solvabilité et d'une liquidité (Christen, Lyman et Rosenberg, 2003).

Également, la régulation impose souvent une transmission régulière (annuelle, trimestrielle ou mensuelle) des rapports pour le suivi des indicateurs prudentiels. C'est un reporting permanent et de qualité qui s'impose aux SFD et demande par conséquent, des ressources humaines de qualité. Ce reporting montrant des signaux positifs par rapport au respect des dispositifs prudentiels permet de dégager une légitimité aux yeux des acteurs externes, de gagner la confiance des investisseurs et de faciliter le financement externe (Rouleau, 2007). Ce financement peut être facilité par l'existence d'un cadre réglementaire et il permettra aux petites et moyennes entreprises (PME) et aux zones rurales d'accéder facilement aux ressources financières (Satta, 2003). 
La régulation prudentielle se fait à travers une réglementation ${ }^{2}$ et une supervision $^{3}$ prudentielle. Ces deux mécanismes ont un caractère prudentiel s'ils permettent de garantir la santé financière des intermédiaires, de prévenir des situations de défaillance et de protéger les petits déposants contre des situations d'insolvabilité et d'illiquidité (Christen et al, 2003 ; Barlet, 2003 ; Hoxhaj, 2010). Une supervision périodique portant sur ces aspects de réglementation permet de contenir les risques auxquels les épargnants sont exposés et d'aiguillonner la profitabilité (Hoxhaj, 2010).

Des travaux en Bolivie, pays pionnier en microfinance, montrent que la régulation prudentielle éloigne les SFD de leur objet social. Elle vise l'adoption de règles standards destinés à stabiliser le secteur et protéger les clients, la commercialisation de la microfinance et privilégie la rentabilité et l'autonomie (Satta, 2004). En effet, les règles prudentielles cherchent à minimiser les risques de perte en protégeant les épargnants, assurent une viabilité aux SFD et in fine, une stabilité du secteur dans son ensemble (Bédécarrats et Marconi, 2009 ; Diani, 2019). La protection des épargnants par un dispositif prudentiel semble vérifier avec l'existence d'un ratio de capitalisation satisfaisant (Tchuigoua et Nekhili, 2012).

La supervision faite par les autorités de régulation et de tutelle permet de veiller au respect des ratios prudentiels et de sanctionner les dérives préjudiciables aux épargnants du secteur financier. La sanction peut aller jusqu'au retrait de l'agrément. A cet effet, il y a un objectif d'inscrire une démarche unique, coercitive et harmonisée permettant aux SFD d'endiguer les risques suivant une même approche et de protéger les épargnants et le secteur.

Cette facette de la régulation peut être expliquée par la théorique néoinstitutionnelle. Selon cette théorie, les pratiques et les formes organisationnelles dépendent de l'environnement dans lequel évoluent les organisations. Ces dernières sont placées dans un environnement hautement institutionnalisé et l'analyse de leur fonctionnement dépasse leurs frontières et fait appel aux institutions ${ }^{4}$ (Landrieux-Kartochian, 2018). Suivant ce déterminisme, Rouleau (2007) parle de pratiques organisationnelles institutionnalisées qui se répètent et font l'objet de routine sous les pressions de l'environnement ${ }^{5}$. Un environnement institutionnel suppose la production de règles, de normes et l'existence d'un dispositif de mise en œuvre des règles

${ }^{2}$ Un ensemble de règles et mesures ayant une force obligatoire adoptées par le législatif et ordonnée par le l'exécutif

${ }^{3}$ Un mode de surveillance externe visant à faire respecter la réglementation

${ }^{4}$ Constituent des règles du jeu destinées à contraindre les organisations à travers des règles formelles et informelles. Pour les institutionnalistes, précurseurs, une institution peut aussi se référer à une organisation, à condition qu'il existe de dirigeants capables d'assurer la promotion de valeurs fortes issues de la communauté.

${ }^{5}$ Il peut être l'état, les organismes réglementaires, les tribunaux, les groupes professionnels, l'opinion publique etc. 
établies (Menard, 2003). Le respect de ces règles conduit à une homogénéité et une similarité sur les pratiques organisationnelles. Il s'agit d'isomorphisme au sens de Dimaggio et Powell (1983) avec trois formes (coercitive, mimétique et cognitive). Les règles coercitives permettent plus d'expliquer le conformisme ou l'isomorphisme auquel les SFD sont soumis par rapport à l'exigence de respect des ratios prudentiels. Elles regroupent les aspects légaux et réglementaires qui s'appliquent aux organisations et les conduisent à adopter un rituel de conformité. Dans des pays comme le Maroc, la régulation prudentielle a permis d'organiser le secteur, homogénéiser les pratiques et assurer la surveillance de la viabilité financière des SFD (Diani, 2019).

Le fonctionnement des SFD au Sénégal est soumis à la réglementation prudentielle à travers la loi $\mathrm{n}^{\circ}$ 2008-47 avec des objectifs clairs à atteindre et une supervision prudentielle faite par la BCEAO et la DRS. Ainsi, les SFD évoluent dans un environnement institutionnel décrit ci-dessus. Une série de ratios sont à respecter afin de protéger les clients et de rendre stable le secteur de la microfinance. Les SFD, selon leur taille et conformément à l'article 44 de ladite loi, sont contraints à déposer, chaque mois ou chaque trimestre, des rapports faisant état de leur conformité aux seuils à atteindre pour les ratios prudentiels de gestion fixés par la BCEAO. Ces contraintes coercitives imposées par la réglementation et la supervision conduisent à un isomorphisme sur le respect des dispositions prudentielles. Ce respect permettrait l'atteinte de la stabilité qui se manifeste par une viabilité et une protection des clients. A l'issue de l'éclairage sur la régulation et sur les pressions coercitives qui en découlent, le concept de stabilité financière est présenté, comme un objectif final de la régulation dans un contexte de microfinance institutionnaliste.

\section{I.2. Stabilité financière comme finalité sous le prisme de l'approche institutionnaliste}

Depuis longtemps, les approches institutionnaliste et welfariste sont analysées comme deux courants qui essayent d'avoir les mêmes objectifs de lutte contre la pauvreté avec des logiques différentes (Noël et Ayayi, 2009). L'approche welfariste est plus adaptée aux ONG qui ne recherchent pas de profit et visent les couches les plus vulnérables pour une distribution exclusive de crédit à des taux très faibles. La rentabilité et le retour sur investissement ne sont pas une priorité. Les entités de financement utilisent des subventions ou des dons et interviennent pour un temps donné et limité. Dans des situations pareilles, la viabilité, la stabilité financière de ces structures ne constituent pas un objectif (Fouda Owoundi, 2010). Une protection des clients n'est pas envisagée car il s'agit, le plus souvent, du crédit direct et par conséquent, une réglementation prudentielle est inopportune (Christen et al, 2003).

Par contre, l'approche institutionnaliste, soutenue par la banque mondiale et le fonds monétaire international, vise les moins pauvres et exige 
une rentabilité sur les crédits injectés car les SFD rémunèrent l'épargne collectée auprès des ménages ou à partir d'emprunts (Noël et Ayayi, 2009). Cette approche, purement commerciale, demande une massification du crédit octroyé qui ne saurait être financé par des subventions et des dons. A cet effet, il serait indispensable de mobiliser d'autres moyens de financement plus volumineux et stables dans le temps (Nzongang, Piot-Lepetit et Kamdem, 2012).

Regardant l'évolution de la microfinance dans le contexte sénégalais avec les phases consolidation et d'intégration, il est évident que les SFD doivent compter sur leurs propres ressources pour couvrir leurs charges d'exploitation. Avec cet objectif de rentabilité, les SFD sont naturellement dans une approche institutionnaliste. L'usage de cette approche est renforcé par l'objectif de stabilité financière du secteur visé dans la loi n 2008-47 et mis en œuvre avec les ratios prudentiels à respecter par tous les SFD.

Les crises financières et l'accélération de l'innovation financière croissante constituent les principales causes d'une quête de stabilité financière (AMAO, 2017). Le secteur de la microfinance a été assaini entre 2008 et 2010, régulé par une nouvelle réglementation et de nouveaux outils prudentiels permettant de garantir la stabilité du secteur.

La pertinence de l'analyse de la stabilité financière dépend toujours d'une période antérieure de difficultés et d'instabilité. Ce qui renseigne sur une situation évolutive soulignant que la stabilité est un état et non une propriété (Abbad et al, 2015).

La stabilité financière décrit une situation dans laquelle les acteurs fonctionnent de manière saine, sans difficultés majeures et avec un bon niveau de performance (BCEAO, 2006). Elle permet une bonne intermédiation financière, avec comme résultante, le renforcement de la confiance des épargnants et la canalisation des ressources collectées vers les projets d'investissement. Elle dépend, entre autres, d'un cadre réglementaire efficace et de supervision prudentielle des institutions (Dannon et Lobez, 2014).

En s'inspirant de la définition d'Illing et Liu (2006) sur la vulnérabilité financière, la stabilité financière, concept opposé, peut être appréciée comme une variable continue avec une série de valeurs dont les points extrêmes renseignent sur des situations de santé financière et de risques faibles. Cette approche d'appréhender la stabilité financière repose sur la démarche de Borio (2009) qui définit la stabilité par la négative, c'est-à-dire l'absence d'instabilité.

Le rapport de l'AMAO (2017) sur la stabilité financière de l'espace CEDEAO fait apparaitre des ratios de stabilité qu'on peut catégoriser en deux types : ratios de solidité financière et de protection des épargnants. Pour la première catégorie, on retrouve les ratios de rentabilité des actifs, de rentabilité 
des capitaux propres et de qualité des actifs (Prêts non performants) (Sorge, 2004).

Les ratios de rentabilité sont normés par la BCEAO et permettent de décrire la capacité des SFD à dégager un résultat pour un investissement donné. Donc, la profitabilité est déjà une nécessité et elle est prise en compte par des ratios d'autosuffisance opérationnelle et de marge bénéficiaire.

Pour la qualité des actifs des SFD, on se réfère impérativement à l'encours de crédit. Il constitue l'actif le plus important pour les SFD. Il permet de générer les produits d'exploitation mais il constitue également une source de perte et de retard de paiement. Deux indicateurs : portefeuille à risque (PAR) et le taux de perte sur créance (TPC) sont combinés afin de capter simultanément les informations sur les pertes et les retards constatés sur les crédits libérés.

Quant aux indicateurs de protection, l'adéquation des fonds propres, la liquidité générale et la liquidité de l'actif sont utilisés dans l'analyse du potentiel des SFD à faire face aux besoins de retrait des épargnants. Ces dimensions de solidité financière (rentabilité et qualité des actifs) et de protection des épargnants sont largement utilisés dans la littérature financière (Albulescu, 2009; Abbad, Achouche et Tadjeddine, 2015) et dans les instructions de la BCEAO de 2010 pour décrire la stabilité financière. Aussi, ils sont qualifiés de ratios centraux et prescrits pour les systèmes financiers (FMI, 2006). Ils sont retenus conformément à leur adaptation et formulation par la BCEAO pour les SFD. L'analyse de la solidité du système financier et des risques susceptibles d'impacter la stabilité financière peut se faire, en partie, avec une approche quantitative et micro prudentielle (BCEAO, 2006). Cette approche micro prudentielle permet de détecter les forces, les faiblesses et les risques de défaillance du système.

Les indicateurs recensés dans la revue, dans les rapports et instructions $\mathrm{n}^{\circ} 10$ et $\mathrm{n}^{\circ} 20$ de la BCEAO seront agrégés pour une compréhension globale et catégorisée pour chaque dimension de la stabilité financière.

L'élaboration d'un indice agrégé est essentielle pour analyser la stabilité financière. En effet, l'usage d'une seule mesure rend difficile la compréhension de la stabilité et par conséquent, un indice synthétique permettrait de mieux décrire la situation (Abbad et al, 2015). Il constitue un outil statistique complémentaire aux modèles économétriques pour rendre compte de la stabilité financière (Rouabah, 2007).

La méthode de pondération permet d'analyser l'apport de chaque dimension, de chaque indicateur à l'indice agrégé. Elle donne également un reflet de la situation de chaque segment du système financier, représenté par une dimension. Selon Illing et Liu (2006), les systèmes de pondération comprennent l'analyse en composante principale, la pondération selon la taille du marché, la pondération à variance égale, et la pondération basée sur la 
fonction de distribution cumulative des variables. Tout système de pondération nécessite une normalisation ${ }^{6}$ des indicateurs avant le regroupement des dimensions pour un indice agrégé.

Cette revue théorique sur la régulation et la stabilité a permis de comprendre l'intérêt de la régulation prudentielle en termes de recherche de stabilité financière des SFD. L'analyse de la stabilité des SFD, après l'adoption d'une réglementation, est faite suivant une méthodologie claire de collecte et de traitement de données.

\section{La méthodologie adoptée}

Pour mieux analyser la stabilité financière des SFD sur la période allant de 2010 à 2015, une base de données de la Direction de la réglementation et de la supervision des SFD (DRS/SFD) 7 de 42 SFD est utilisée. Cette base permet de calculer tous les indicateurs mobilisés dans l'analyse de la rentabilité, de la qualité des actifs et de la protection des clients.

Pour répondre à la question de recherche, un IASF et des sous indices pour chaque dimension de la stabilité financière sont élaborés. L'analyse de ces indices dans le temps permet de conclure sur la stabilité globale après réforme et aussi sur le comportement des SFD par rapport à des aspects décisifs de solidité financière et de protection des épargnants.

La construction de l'IASF nécessite le respect des étapes suivantes : justification théorique des dimensions et indicateurs, la normalisation, la pondération et l'agrégation.

\section{a. Justification théorique du choix des dimensions et indicateurs}

$\mathrm{Au}$ regard des développements théoriques sur la stabilité financière notés sur la partie (I.2), le tableau $\mathrm{n}^{\circ} 2$ synthétise les dimensions et les indicateurs utilisés pour établir un IASF.

6 Plusieurs méthodes de normalisation existent (statistique, empirique, axiologique et mathématique) et le choix d'une option nécessite la prise du nombre et du type de variable (qualitative ou quantitative) (Albulescu, 2009)

${ }^{7}$ La DRS est une direction du ministère de finance qui assure la tutelle. La DRS reçoit à la fin de chaque année les états financiers des SFD agréés et aussi de façon périodique les rapports de suivi sur le respect des ratios prudentiels de gestion. Elle a comme mission également d'établir des rapports trimestriels, annuels et des statistiques sur le secteur de la microfinance au Sénégal 
Tableau 2 : Indicateurs retenus pour l'IASF

\begin{tabular}{|c|c|c|c|c|}
\hline Indice & Dimensions & Ratios & Seuils & ité des ratios \\
\hline \multirow{9}{*}{ IASF } & \multirow{4}{*}{ Rentabilité } & Autosuffisance opérationnelle (OA) & $>130 \%$ & \multirow{6}{*}{$\begin{array}{l}\text { Ils permettent de déceler } \\
\text { l'aptitude à être profitable } \\
\text { par rapport à un } \\
\text { investissement. Aussi, les } \\
\text { deux derniers indicateurs } \\
\text { permettent d'analyser la } \\
\text { qualité de portefeuille de } \\
\text { crédit et les risques encourus } \\
\text { sur l'encours de crédit, } \\
\text { l'actif sur le plus important } \\
\text { des SFD }\end{array}$} \\
\hline & & Rentabilité économique (RE) & $>3 \%$ & \\
\hline & & Rentabilité financière (RF) & $>15 \%$ & \\
\hline & & Taux de marge bénéficiaire (TMB) & $>20 \%$ & \\
\hline & \multirow{2}{*}{$\begin{array}{l}\text { Qualité des } \\
\text { actifs }\end{array}$} & Portefeuille à risque à $90 \mathrm{~J}$ (PAR) & $<3 \%$ & \\
\hline & & Taux de perte sur créance (TPC) & $<2 \%$ & \\
\hline & \multirow{3}{*}{$\begin{array}{l}\text { Protection } \\
\text { des clients }\end{array}$} & Liquidité de l'actif (LA) & $>5 \%$ & \multirow{3}{*}{$\begin{array}{l}\text { Ils signalent le caractère } \\
\text { liquide des actifs et } \\
\text { solvables du SFD. Ils } \\
\text { décrivent la capacité des } \\
\text { SFD à couvrir les besoins } \\
\text { de retrait des clients }\end{array}$} \\
\hline & & Liquidité générale (LG) & $>80 \%$ & \\
\hline & & Taux de capitalisation (TC) & $>15 \%$ & \\
\hline
\end{tabular}

Source: Instructions 10 et 20 de la BCEAO ; Albulescu, 2009 ; Abbad et al, 2015 ; FMI, 2006

\section{b. Normalisation}

Dans ce travail, la méthode de normalisation «Min-Max » (méthode statistique), très répandue dans la littérature de création d'indice, est retenue. Pour l'analyse de la robustesse des résultats, une autre méthode, le Soft-max, est utilisée.

Soit $x_{q c}^{t}$ la valeur de l'indicateur q pour le SFD c à la date t et $\underline{c}$ le SDF de référence. La méthode du Min-Max est donné par :

$$
I_{q c}^{t}=\frac{x_{q c}^{t}-\min _{c}\left(x_{q}^{t_{0}}\right)}{\max _{c}\left(x_{q}^{t_{0}}\right)-\min _{c}\left(x_{q}^{t_{0}}\right)}
$$

Pour prendre en compte la cohérence temporelle dans le calcul de l'indice, la normalisation des panels est d'abord faite. Ensuite, les valeurs minimales et maximales de chaque indicateur sont calculées pour les individus et les périodes. La transformation donne :

$$
I_{q c}^{t}=\frac{x_{q c}^{t}-\min _{t \in T} \min _{c}\left(x_{q}^{t}\right)}{\max _{t \in T} \max _{c}\left(x_{q}^{t}\right)-\min _{t \in T} \min _{c}\left(x_{q}^{t}\right)}
$$

\section{c. La pondération et l'agrégation}

L'agrégation et la pondération ont pour rôle de déterminer le poids de chaque indicateur dans le calcul de l'indice. Ici, une technique d'analyse en composantes principales en panel (PACP) est retenue. Ce choix est justifié par le fait qu'il possible de résumer un ensemble de variables sans perdre 
l'importante variabilité des données originales. De plus, avec la dimension de panel, cette technique permet de prendre en compte l'évolution de l'indice dans le temps. L'objectif du PACP est d'expliquer la variance des données observées par quelques combinaisons linéaires des données d'origine.

Dans une situation de panel, un vecteur de données multidimensionnel est présenté :

$$
X_{T \times Q}=\left(x_{1}^{t}, x_{2}^{t}, \ldots, x_{Q}^{t}\right), t \in T
$$

Avec $\mathrm{t}$ la dimension temporelle et $\mathrm{Q}$ le nombre de variables.

Soit $\Sigma_{Q \times Q}$ la matrice de corrélation des variables $X_{Q \times T}$. La composante principale $Z_{i}^{t}, i=1,2, \ldots, Q$ est définie comme suit :

$$
\begin{gathered}
\left\{Z_{1}^{t}=a_{11} x_{1}^{t}+a_{12} x_{2}^{t}+\cdots+a_{1 Q} x_{Q}^{t} Z_{2}^{t}=a_{21} x_{1}^{t}+a_{22} x_{2}^{t}+\cdots+a_{2 Q} x_{Q}^{t} \cdots Z_{Q}^{t}\right. \\
=a_{Q 1} x_{1}^{t}+a_{Q 2} x_{2}^{t}+\cdots+a_{Q Q} x_{Q}^{t}
\end{gathered}
$$

Sous forme matricielle, $Z=A^{\prime} X_{Q \times T}$, où $A=\left(a_{1}, a_{1}, \ldots, a_{Q}\right)$, coefficient de $A$ qui minimise la variance de $Z=E\left(Z Z^{\prime}\right)=A^{\prime} \Sigma A$ soumis aux contraintes suivantes :

$$
a_{1}^{\prime} a_{1}=a_{2}^{\prime} a_{2}=\cdots=a_{Q}^{\prime} a_{Q}=1 \text { et } \operatorname{cov}\left(a_{i}^{\prime} x, a_{j}^{\prime} x\right)=0, i \neq j
$$

La solution au problème des valeurs propres et des vecteurs propres résultant de ce programme d'optimisation est $\lambda_{i}$ qui est égale à la variance de $Z$ avec $\lambda_{1}>\lambda_{2}>\cdots>\lambda_{Q}$.

Les facteurs obtenus à partir du PACP peuvent maintenant être utilisés pour calculer les différents poids. Dans un premier temps, le PACP est appliqué sur les variables dans chaque dimension pour obtenir les différents poids. Une fois les poids obtenus, le PACP est appliqué, dans un second temps, aux sous-indices pondérés pour établir l'indice global. A partir de là, les résultats sur l'IASF seront présentés et par rapport à chacune de ses dimensions.

Avec l'approche min-max utilisée, les valeurs extrêmes peuvent fausser la distribution des valeurs normalisées. Pour éviter ce problème, la méthode softmax est mobilisée pour tester la robustesse des résultats. L'un des avantages de cette technique est sa capacité à réduire l'influence des valeurs extrêmes ou des valeurs aberrantes. La normalisation est donnée par :

Avec

$$
I_{q c}^{t}=\frac{1}{1+\exp (-v)}
$$

$$
v=\frac{\left[x_{q c}^{t}-\underline{x}_{q c}\right]}{\sigma_{q c}}
$$

$\underline{x}_{q c}$ représente la moyenne de l'indicateur et $\sigma_{q c}$ son écart type. 


\section{Résultats et discussions}

Il est présenté dans cette partie la contribution de la rentabilité, de la qualité des actifs et la protection des clients à l'élaboration de l'IASF.

Aussi, l'évolution temporelle de l'IASF et des sous indices sera analysée pour conclure sur la solidité financière, la protection des clients et sur la stabilité financière globale des SFD.

\section{III.1. Contribution des indicateurs et des dimensions à l'élaboration d'indices}

Les principaux résultats liés à l'analyse en composantes principales en panel sont résumés dans le tableau $\mathrm{n}^{\circ} 3$.

Tableau 3 : Résultats sur l'agrégation et les poids des indices

\begin{tabular}{|c|c|c|c|c|c|c|c|c|c|c|c|c|}
\hline \multicolumn{13}{|c|}{ Nombre de composantes principales (Min-Max) } \\
\hline & \multicolumn{4}{|c|}{ Rentabilité } & \multicolumn{2}{|c|}{$\begin{array}{l}\text { Qualité des } \\
\text { actifs }\end{array}$} & \multicolumn{3}{|c|}{$\begin{array}{l}\text { Protection des } \\
\text { clients }\end{array}$} & \multirow[b]{2}{*}{1} & \multirow{2}{*}{$\frac{\text { IASF }}{2}$} & \multirow[b]{2}{*}{3} \\
\hline & 1 & 2 & 3 & 4 & 1 & 2 & 1 & 2 & 3 & & & \\
\hline Prop. & 0.52 & 0.34 & 0.09 & 0.04 & 0.63 & 0.37 & 0.52 & 0.32 & 0.15 & 0.52 & 0.27 & 0.21 \\
\hline Cum. & 0.52 & 0.86 & 0.96 & 1.00 & 0.63 & 1.00 & 0.52 & 0.85 & 1.00 & 0.52 & 0.79 & 1.00 \\
\hline \multicolumn{13}{|c|}{ Loadings au carré } \\
\hline Variables & $\mathrm{AO}$ & RE & RF & TMB & PAR & TPC & LA & LG & TC & Rentabilité & Qualité & Protection \\
\hline F1 & 0.43 & 0.20 & 0.02 & 0.35 & 0.50 & 0.50 & 0.05 & 0.46 & 0.48 & 0.40 & 0.29 & 0.31 \\
\hline $\mathrm{F} 2$ & 0.00 & 0.31 & 0.59 & 0.10 & 0.50 & 0.50 & 0.94 & 0.04 & 0.01 & 0.00 & 0.56 & 0.42 \\
\hline \multicolumn{13}{|c|}{ Pondération } \\
\hline Poids & 0.26 & 0.25 & 0.24 & 0.25 & 0.50 & 0.50 & 0.40 & 0.30 & 0.30 & 0.26 & 0.39 & 0.35 \\
\hline
\end{tabular}

Source : Résultats des analyses en composantes principales à partir des données de la base de la DR

$\mathrm{Au}$ regard des résultats ci-dessus, la contribution des variables pour chaque dimension et aussi l'apport des dimensions à l'élaboration de l'IASF sont exposés. Pour la dimension rentabilité, les variables (OA, RE, RF, TMB) ont pratiquement le même niveau de contribution. Il en est de même des variables PAR et TPC pour la dimension «Qualité des actifs ».

Cependant, la variable ou l'indicateur « liquidité de l'actif » contribue plus à la «protection des clients». La liquidité générale et le taux de capitalisation ont le même niveau de contribution à la protection des clients épargnants.

Pour l'indice agrégé, la solidité financière, appréciée à partir de la rentabilité et la qualité des actifs, contribue plus à l'élaboration de l'IASF. Ces deux dimensions expliquent à $65 \%$ la stabilité financière avec une contribution plus importante (39\%) de la qualité des actifs.

La protection des clients épargnants contribue à $35 \%$ à la stabilité financière des SFD. 


\section{III.2. Appréciation de la stabilité financière}

Les différentes tendances de la solidité financière (rentabilité et qualité des actifs), de la protection des clients et de la stabilité financière des SFD sont appréciées respectivement à partir des sous indices et de l'IASF. Elles sont analysées à partir des graphiques agrégés ci-dessous.

Figure ${ }^{\circ} 1$ : Résultats sur l'évolution de la stabilité financière des SFD
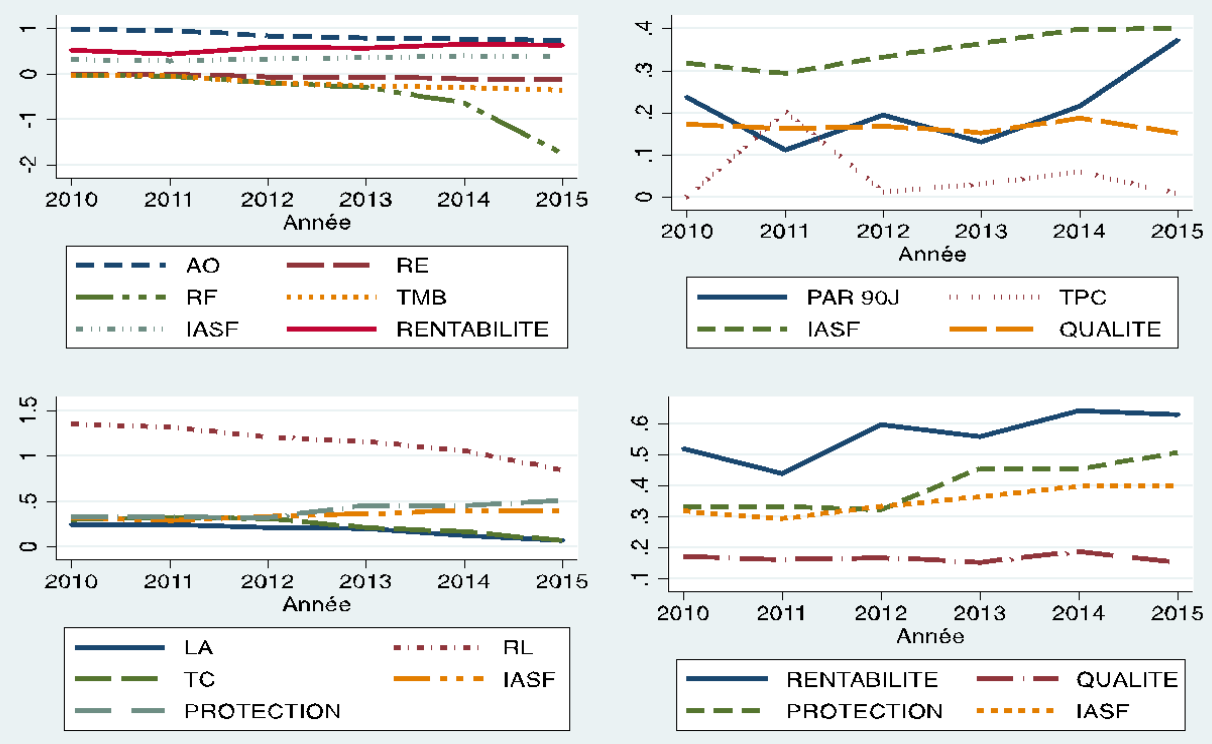

Source: Résultats issus du traitement des données de la base de la DRS

Pour la solidité financière, une évolution stable de ses déterminants est notée. Les résultats affichent une évolution constante de la rentabilité de 2010 à 2015. Cependant les seuils fixés par la BCEAO ne sont pas atteints pour les ratios comme $\mathrm{OA}, \mathrm{TMB}$ etc. La rentabilité financière a connu une chute et est devenue négative, à partir de 2012. Les SFD ont des difficultés, en moyenne, pour dégager suffisamment de produits pour la couverture des charges. La viabilité financière des SFD peut être améliorée par une maximisation du volume de crédit, une fixation du taux d'intérêt à niveau suffisamment rémunérateur et une maitrise du PAR (Diop, 2013).

Quant à la qualité des actifs, une évolution stable est constatée, juste après l'adoption de la nouvelle régulation. Cependant, en 2013, le PAR a augmenté jusqu'à dépasser le seuil de $3 \%$ et permet de noter des retards et des impayés de plus de 90 jours. La stabilité de la qualité des actifs est due certainement à une relative maîtrise du taux de perte sur créances (TPC), à partir de 2011.

Des recherches ont montré que le PAR peut être amélioré par la fourniture de crédit correspondant juste au besoin (Honlonkou, Acclassato, et Quenum, 2001 et Lanha, 2002), la formalisation des petites entreprises 
(Tressel, 2003 ; Gregory et Tenev, 2001), l'expérience en terme d'années d'existence du débiteur (Ben Soltane, 2008 ; Matabaro et Mugisho, 2013), une ligne unique de crédit (Morduch, 2000) et l'existence de garantie mobilière (Blazy et Weill, 2013).

Globalement, on note une évolution stable de la solidité financière à travers la rentabilité et la qualité des actifs. Néanmoins, des efforts sont à faire pour améliorer la profitabilité et diminuer les retards et les impayés. Les seuils fixés par la BCEAO pour les ratios de rentabilité et de PAR doivent être atteints afin de sortir les SFD de la zone stabilité précaire.

En ce qui concerne la dimension "protection des clients», une tendance haussière est notée depuis 2012. Ce qui montre une prise en compte des besoins des épargnants et une diminution des risques auxquels ils sont exposés. Au regard de l'évolution des courbes du ratio de liquidité (RL), de liquidité de l'actif (LA) et de taux de capitalisation (TC), les seuils fixés par la BCEAO sont pratiquement atteints. Cependant, une baisse est constatée depuis 2010. Des mesures de correction doivent être prises afin de redresser cette tendance qui pourra, à terme, exposer les épargnants à des difficultés de retrait. Un regard critique doit être porté sur la liquidité des actifs qui contribue à plus de $40 \%$ à la protection des épargnants.

Pour une synthèse sur l'IASF, une tendance positive est constatée depuis 2010. De manière générale, une amélioration est notée depuis l'entrée en vigueur de la nouvelle régulation prudentielle. La consolidation et l'amélioration de cette stabilité financière dépendront d'efforts additionnels sur la rentabilité et sur le PAR.

Également, la liquidité des actifs est un point important à surveiller afin que la contribution de la dimension «protection des épargnants » puisse se consolider et s'améliorer pour une meilleure stabilité financière.

Le poids $(65 \%)$ des deux dimensions (rentabilité et qualité des actifs) souligne le caractère indispensable d'une solidité financière dans la quête d'une stabilité financière du secteur de la microfinance. Mieux, les dimensions prises individuellement montrent que la stabilité dépend de la qualité des actifs (39\%) et de la protection des épargnants (35\%) (Cf tableau 3).

\section{III.3. Analyse de la robustesse des résultats}

Ce paragraphe permet d'analyser la robustesse des résultats présentés dans la figure $\mathrm{n}^{\circ} 2$. 
Figure $\mathbf{n}^{\circ} 2$ : Résultats du test de robustesse par la méthode Soft Max
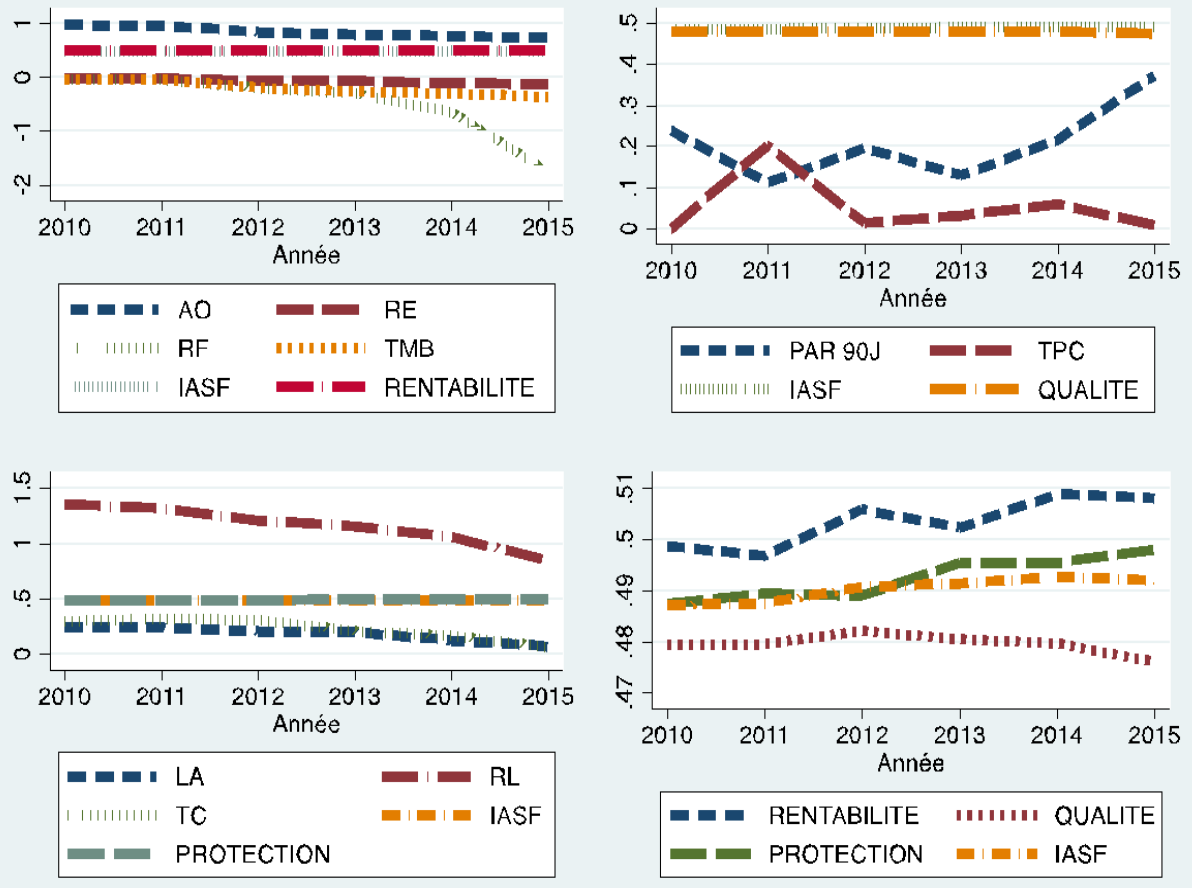

Source : Résultats issus du traitement des données de la base de la DRS

L'analyse comparée de la figure 1 et de la figure 2 montre les mêmes tendances sur la rentabilité, la qualité des actifs, la protection des épargnants et la stabilité globale des SFD. Cependant, les résultats du test de robustesse précisent que la qualité des actifs est moins bonne avec un niveau très élevé de la courbe. La méthode Soft Max a permis de corriger les valeurs extrêmes (nulle) du PAR constatées sur la base de données. Cette insuffisance notée dans la qualité du portefeuille confirme la situation sectorielle décrite dans les rapports de la DRS en 2018 et 2019.

Globalement, les résultats sont robustes et correctes à l'utilisation d'autres méthodes de normalisation et de pondération.

\section{Conclusion}

Les résultats notés à partir de la courbe des sous indices et de l'IASF montrent une évolution constante de la rentabilité et de la qualité des actifs.

Cependant, cette situation peut être améliorée car cette stabilité ne correspond pas toujours à l'atteinte des seuils fixés par la BCEAO sur les ratios prudentiels. En effet, les ratios de rentabilité dénotent une insuffisance de produits d'exploitation pour la couverture des charges d'exploitation. Aussi, 
la qualité des actifs mérite une attention particulière avec un portefeuille à risque (PAR) qui dépasse le seuil de 3\%, à partir de 2012.

Les SFD du secteur doivent améliorer leur gestion afin de générer plus de marge d'exploitation et de rendre la tendance haussière pour la rentabilité et baissière pour le PAR et la qualité des actifs.

Quant à la dimension «protection des clients », la situation est plus favorable. Elle contribue à hauteur de $35 \%$ à la stabilité financière. Une situation de plus en plus satisfaisante est notée, à partir de 2012. Sur toute la période d'analyse, les seuils semblent atteints pour tous les indicateurs malgré une tendance régressive des ratios de liquidité, de liquidité de l'actif et de taux de capitalisation.

Une agrégation des résultats permet de se prononcer sur l'IASF qui souligne une évolution stable, légèrement positive de la stabilité financière des SFD, depuis 2010.

Cependant, la profitabilité, le PAR et la liquidité de l'actif méritent une surveillance régulière afin d'augmenter les produits d'exploitation, de minimiser les retards, les impayés et d'avoir plus de ressources financières disponibles. La disponibilité d'une base de données plus récente permettrait d'actualiser les résultats et de donner plus de valeur ajoutée.

Le travail pourrait être prolongé sur la recherche des déterminants de la liquidité de l'actif des SFD. Cette variable est capitale et contribue à hauteur de $40 \%$ à la protection des épargnants.

\section{References:}

1. Abbad, H., Achouche, M. \& Tadjeddine, Y. (2015). Evaluation du système financier Algérien : Construction d'un indice agrégé de stabilité bancaire. Working Paper P25. http://economix.fr.

2. Albulescu, C.T. (2009). Forecasting Romanian Financial System Stability Using a Stochastic Simulation Model. Working Paper 2009.4, International Network for Economic Research, $p 22$.

3. Bédécarrats, F. \& Marconi, R. (2009). L'influence de la régulation sur la contribution de la microfinance au développement : le cas de la Bolivie. Revue Tiers Monde, Vol. 50, No. 197 (JANVIER-MARS 2009), pp. 71-90.

4. Ben Soltane, B. (2008). Déterminants de la durée de retard de remboursement des microcrédits individuels: application d'un modèle de durée. African Review of Money Finance and Banking, (2008), pp, 91-116.

5. Blazy, R. \& Weill, L. (2013). Why do banks ask for collateral in SME lending?. Applied financial economy, Volume 23,2013-Issue 13. pp $1109-1122$ 
6. Borio, C. (2009). L'approche macroprudentielle. Revue de la stabilité financière ${ }^{\circ} 13$, pp. 35-46.

7. BEAC (Banque des Etats de l'Afrique Centrale). 2016. La stabilité financière en Afrique centrale. Revue de la stabilité financière en Afrique centrale pp 51-58

8. Dannon, P. H. \& Lobez, F. (2014). La régulation bancaire dans l'union économique et monétaire ouest-africaine est-elle efficace ? Revue d'économie financière, $N^{\circ} 116$, pp 279-304.

9. Diani, A. (2019). Logique d'acteurs et régulation dans le champ de la microfinance marocaine : vers une commercialisation croissante. RECMA, 2019/4 N³54, pp 104-119

10. Dimaggio, P. J. \& Powell, W. W. (1983).The iron cage revisited: institutional isomorphism and collective rationality in organizational fields. American Sociological Review, vol. 48, pp. 147-160.

11. Diop, C. M. (2013). L'analyse des déterminants de la viabilité financière des structures financières décentralisées (SFD) au Sénégal. African Management Studies (AMS), 1(2), PP. 125-160. http://www.samuniversity.org/index.php?journal=AMS\&page=issue \&op=archive

12. Fouda Owoundi, J.P. (2010). La réglementation a-t-elle un impact sur les performances de la microfinance? Le cas du Cameroun. African Review of Money Finance and Banking, (2010), pp. 29-58.

13. Gregory, N. \& Tenev, S. (2001). The Financing of Private Enterprise in China. Finance and Development 38, pp. 14-17.

14. Honlonkou, A. N., Acclassato, D. H. \& Quenum, C.V.C. (2001). Problématique de remboursement des crédits dans les systèmes financiers décentralisés et garantie de prêts aux petits opérateurs économiques au Bénin. Cahier de Recherche ELIFID 00-2, P 61

15. Hoxhaj, R. (2010). Regulation and supervision of microfinance in Albania. BEH - Business and Economic Horizons, Issue 2, July 2010, pp. $75-81$.

16. Illing, M. \& Liu, Y. (2006). Measuring financial stress in a developed country: An applicationto Canada. Journal of Financial Stability, Vol. 02, Issue 3, pp 243-265.

17. Landrieux-Kartochian, S. (2018). Théorie des organisations, $4^{\text {ième }}$ édition. (pp : 95-124). Gualino 70, rue du Gouverneur Général Éboué 92131 Issy-les-Moulineaux cedex.

18. Lanha, M. (2002). Résolution des problèmes d'information en microfinance Analyse à partir de la stratégie de Vital-Finance Bénin. Mondes en développement 2002/3 (no 119), p, 47-62. 
19. Matabaro, B. L. \& Mugisho, M.G. (2013). Analyse des déterminants et de la probabilité de défaut de remboursement de crédit dans les IMF d'uvira : cas de mecre uvira. Journal of economics and social sciences, $\mathrm{N}^{\circ} 2$, pp 41-62

20. Menard, C. (2003). L'approche néo institutionnelle: des concepts, une méthode, des résultats. Cahier d'économie politique, 2003/1 $n^{\circ} 44, p p$ $103-118$.

21. Ellé, S.M. (2017). Microcrédits et performances financière et sociale des institutions de microfinance au Cameroun. La revue gestion et organisation, Volume 9, Numéro 2, pp 79-91

22. Morduch, J. (2000). The Microfinance Schism. World Development, Vol, 28, No, 4, pp, 617-629.

23. Noël, C. \& Ayayi, A. (2009). L'autosuffisance des institutions de microfinance est-elle une nécessité ?. Humanisme et Entreprise, $n^{\circ}$ 292, pp 65 à 75 .

24. Nzongang, J., Piot-Lepetit, I. \& Kamdem, D. (2012). La mesure de l'efficacité financière et sociale des institutions de microfinance du réseau $\mathrm{MC}^{2}$ au Cameroun. Mondes en développement, $n^{\circ} 160$, pp 99 à 116.

25. Ory J.N., Jaeger M. \& Gurtner E. (2006). La banque à forme coopérative peut-elle soutenir durablement la compétition avec la banque SA ? Finance Contrôle Stratégie, vol. 9, $n^{\circ}$ 2, p. 121-157.

26. Rouabah, A. (2007). Mesure de la vulnérabilité du secteur bancaire luxembourgeois. Working paper $N^{\circ} 24$. Banque Centrale Luxembourg, pp 1-20.

27. Rouleau, L. (2007). Théories des organisations : Approches classiques, contemporaines et de l'avant-garde. Presses de l'université de Quebec.

28. Satta, T. A. (2004). Microfinance regulation influence on small firms' financing in Tanzania. Financial Regulation and Compliance Volume $12, n^{\circ} 1, p p$ 64-74.

29. Sorge, M. (2004): Stress-testing financial systems: an overview of current methodologies. BIS Working papers, $n^{\circ} 165$, December, $p 41$

30. Tchuigoua, H.T. \& Nekhili M. (2012). Gestion des risques et performance des institutions de microfinance. Revue d'économie industrielle, 138, 2ème trimestre 2012, pp 127-148

31. Tressel, T. (2003). Dual financial systems and inequalities in economic development. Journal of Economic Growth, Vol, 8, No, 2 (Jun,, 2003), pp 223-257. 


\section{Rapports ou Archives}

32. Albulescu, C. T. (2008). La dynamique de la stabilité du système financier roumain : une analyse en termes d'indice agrégé de stabilité. Munich Personal RePEc Archive, Online at https://mpra.ub.unimuenchen.de/16754.

33. AMAO (2017). Evolution et stabilité du secteur financier au sein de la CEDEAO

34. Barlet, K. (2003). Le point sur la réglementation et la supervision de la microfinance. BIM $\mathrm{n}^{\circ} 30$ septembre 2003. CGAP, http://www.cgap.org/.

35. BCEAO (2001). Etude sur la viabilité financière des SFD. Monographies de la BCEAO www.bceao.int

36. BCEAO (2006). Revue de la stabilité financière dans l'union économique et monétaire ouest africaine

37. BCEAO (2010). Instruction $\mathrm{n}^{\circ} 010-08-2010$ relative aux règles prudentielles applicables aux structures financières décentralisées des Etats membres de l'union monétaire ouest Africaine (UMOA).https://www.bceao.int/sites/default/files/inlinefiles/chapitre_7_ _reglementation_specifique_aux_institutions_de_microfinance.pdf

38. BCEAO (2010). Instruction $\mathrm{n}^{\circ} 20-12-2010$ relative aux indicateurs périodiques à transmettre par les systèmes financiers décentralisés au ministère en charge des finances, à la banque centrale et à la commission bancaire de l'Union Monétaire Ouest Africaine (UMOA). https://www.bceao.int/sites/default/files/inline-files/chapitre_7_ _reglementation_specifique_aux_institutions_de_microfinance.pdf

39. Christen, R. P., Lyman, T. R. \& Rosenberg, R. (2003). Principes directeurs en matière de réglementation et de supervision de la microfinance. CGAP/le Groupe de la Banque mondiale, Washington, D.C. 20433 Etats-Unis.

40. Direction de la réglementation et de la supervision des systèmes financiers décentralisés DRS/SFD. (2008). Loi $\mathrm{N}^{\circ} 2008-47$ du 03 septembre 2008 portant réglementation des systèmes financiers décentralisés (SFD) au Sénégal. https://drssfd.gouv.sn/sitedrs/index.php/2017/12/26/loi-2008-47/

41. Direction de la réglementation et de la supervision des systèmes financiers décentralisés (DRS/SFD). (2019). Note sur la situation des SFD, trimestre 1 et 2 . https://drs-sfd.gouv.sn/sitedrs/wpcontent/uploads/2019/06/Note-sur-la-situation-des-SFD-au-1ertrimestre-2019.pdf

42. Direction de la réglementation et de la supervision des systèmes financiers décentralisés (DRS/SFD). (2018). note sur la situation des 
SFD, (trimestres $1 ; 2 ; 3$ et 4 ). https://drs-sfd.gouv.sn/sitedrs/wpcontent/uploads/2019/06/Note-sur-la-situation-des-SFD-au-1ertrimestre-2019.pdf

43. FMI. (2006). Indicateurs de solidité financière. Guide d'établissement. http://www.imf.org

44. Loi $\mathrm{N}^{\circ} 2008-47$ du 03 septembre 2008 portant réglementation des systèmes financiers décentralisés (SFD) au Sénégal. https://drssfd.gouv.sn/sitedrs/index.php/2017/12/26/loi-2008-47/

45. OCDE. (2010). Renforcer la stabilité financière en améliorant la réglementation. Etudes économiques de l'OCDE, $\mathrm{N}^{\circ} 2$ pp 95-130

46. SAKHO D. (2004). Viabilité et lutte contre la pauvreté. Rapport publié par le ministère des petites et moyennes entreprises, de l'entreprenariat féminin et de la microfinance du Sénégal, www.gtms.sn/ IMG/pdf/Rapport_provisoire_VLCP.pdf 